\title{
Le problème des relations amour-haine-indifférence
}

\author{
Paul Franceschi \\ Université de Corse \\ http://www.univ-corse.fr/ franceschi
}

Article publié dans Semiotica (2005), volume 152 (1-4), pages 251-260

\begin{abstract}
Dans Franceschi (2002), j'ai présenté une théorie basée sur les matrices de concepts qui se propose de constituer une alternative à la classification proposée par Greimas, dans le domaine de l'analyse paradigmatique. Le problème de la détermination des relations existant entre les concepts d'amour, de haine et d'indifférence, se pose dans cette construction. Je m'attache ainsi à décrire le problème des relations amour-haineindifférence de manière détaillée, ainsi que plusieurs solutions qui ont été proposées pour le résoudre. Je décris enfin une solution à ce problème, basée sur une extension de la théorie des matrices de concepts.
\end{abstract}

Dans ce qui suit, je m'attacherai à fournir une solution au problème posé par la définition des relations entre les concepts suivants: amour, haine et indifférence. Je décrirai tout d'abord le problème de manière détaillée ainsi que plusieurs solutions qui ont été proposées auparavant. Je présenterai ensuite ma propre analyse concernant ce problème, basée sur une extension de la théorie des matrices de concepts.

\section{Le problème}

Le problème est celui de la définition correcte des relations existant entre les concepts d'amour, de haine et d'indifférence. Appelons AHI un tel problème. Dans Franceschi (2002), j'ai décrit une théorie basée sur les matrices de concepts qui se propose de constituer une alternative à la classification proposée par Greimas, dans le domaine de l'analyse paradigmatique. Le problème AHI constitue une difficulté dans le cadre de cette construction et il s'avère ainsi nécessaire d'analyser un tel problème avec précision. Quelles sont donc les relations précises qui existent entre ces trois concepts? De prime abord, la définition des relations entre l'amour et la haine apparaît évidente. Ces concepts sont des contraires. On peut penser ici que la définition d'une telle relation devrait être consensuelle. Toutefois, le problème surgit lorsqu'on considère le rapport existant entre, d'une part l'amour et l'indifférence, et d'autre part la haine et l'indifférence. Dans ces derniers cas, aucune réponse ne s'impose comme évidente.

Cependant, il s'avère utile de clarifier les données du problème. Car dans le présent contexte, qu'attendons-nous donc d'une solution au problème AHI? De fait, une solution rigoureuse se doit de définir avec précision les trois relations $\mathrm{R}, \mathrm{S}, \mathrm{T}$ telles que amour $\mathrm{R}$ haine, amour $\mathrm{S}$ indifférence et 
haine $\mathrm{T}$ indifférence. Et les définitions de ces dernières relations doivent être aussi précises que possible.

Il convient de mentionner ici que plusieurs auteurs ont énoncé le problème AHI et ont également proposé une solution. On peut souligner en particulier que les difficultés présentées dans le cadre du calcul propositionnel par des affirmations du type $x$ aime $y$, ou $x$ hait $y$, ou $x$ est indiffèrent à $y$ ont été mentionnées par Emile Benzaken (1990: 63):

La difficulté cependant peut provenir de paires de mots dont l'un exprime le contraire (négation) de l'autre; "haïr" peut être pris comme la négation forte de "aimer" tandis que "être indifférent" en serait la négation faible.

L'auteur y expose le problème des relations amour-haine-indifférence et propose sa propre solution: la haine est la négation forte de l'amour, et l'indifférence est la négation faible de l'amour.

Cependant, la solution de Benzaken ne s'avère pas entièrement satisfaisante, pour les raisons suivantes. En premier lieu, cette façon de résoudre le problème définit les relations entre l'amour et la haine (négation forte, selon l'auteur) et entre l'amour et l'indifférence (négation faible), mais ne définit pas précisément les relations existant entre l'indifférence et la haine. Il y a une lacune, une absence de réponse à ce stade. Car on l'a vu, une solution satisfaisante au problème AHI se doit d'élucider la nature des relations entre les trois concepts. En second lieu, il apparaît que la différence entre la négation faible et la négation forte n'est pas explicitée dans la solution présentée par Benzaken. Pour ces deux raisons, cette dernière solution pour le problème $\mathrm{AHI}$ ne se révèle pas satisfaisante.

Dans un contexte très différent, Rick Garlikov (1998) met l'accent sur des difficultés de même nature que celles soulignées par Benzaken:

In a seminar I attended one time, one of the men came in all excited because he had just come across a quotation he thought very insightful - that it was not hate that was the opposite of love, but that indifference was the opposite of love, because hate was at least still an emotion. (...) I pointed out to him that both hate and indifference were opposites of love, just in different ways, that whether someone hated you or was indifferent toward you, in neither case did they love you.

Garlikov décrit ainsi le problème des relations amour-haine-indifférence et propose implicitement une solution de nature semblable à celle fournie par Benzaken. Pour cette raison, la solution décrite par Galikov souffre des mêmes défauts que ceux présentés par la solution de Benzaken.

Dans ce qui suit, afin de préparer les étapes vers une solution au problème AHI, je m'attacherai tout d'abord à mettre en place le cadre conceptuel approprié.

\section{Un cadre conceptuel}

J'esquisserai ici le cadre formel qui a été décrit de manière détaillée dans Une classe de concepts (2002). Considérons tout d'abord une dualité donnée. Dénotons-la par A/Ā. A ce stade, A et $\bar{A}$ sont des concepts duaux. A et $\overline{\mathrm{A}}$ peuvent en outre être considérés comme des concepts qui présentent une composante contraire $c \in\{-1,1\}$ dans une dualité $\mathrm{A} / \overline{\mathrm{A}}$, et tels que $c[\mathrm{~A}]=-1$ et $c[\overline{\mathrm{A}}]=1$. On peut considérer également que $A$ et $\bar{A}$ sont des concepts neutres qui peuvent être ainsi dénotés par $\mathrm{A}^{0}$ et $\overline{\mathrm{A}}^{0}$.

A ce stade, nous sommes en mesure de définir la classe des pôles canoniques. Considérons l'extension de la classe précédente $\left\{\mathrm{A}^{0}, \overline{\mathrm{A}}^{0}\right\}$, telle que $\mathrm{A}^{0}$ et $\overline{\mathrm{A}}^{0}$ admettent respectivement un concept corrélatif positif et négatif. De tels concepts possèdent un certain support intuitif. Dénotons-les respectivement par $\left\{\mathrm{A}^{+}, \mathrm{A}^{-}\right\}$et $\left\{\overline{\mathrm{A}}^{+}, \overline{\mathrm{A}}^{-}\right\}$. À ce stade, pour une dualité donnée $\mathrm{A} / \overline{\mathrm{A}}$, on obtient les concepts suivants: $\left\{\mathrm{A}^{+}, \mathrm{A}^{0}, \mathrm{~A}^{-}, \overline{\mathrm{A}}^{+}, \overline{\mathrm{A}}^{0}, \overline{\mathrm{A}}^{-}\right\}$. Appelons-les les pôles canoniques. On peut noter ici qu'on pourrait employer de manière alternative la notation $\alpha(\mathrm{A} / \overline{\mathrm{A}}, c, p)$ pour un pôle canonique. ${ }^{1}$ Dans tous les cas, les composants d'un pôle canonique sont une dualité $\mathrm{A} / \overline{\mathrm{A}}$, une composante contraire $c \in\{-1$, $1\}$ et une polarité canonique $p \in\{-1,0,1\}$. Cette définition des pôles canoniques conduit à distinguer

\footnotetext{
${ }^{1}$ Avec cette dernière notation, la matrice des pôles canoniques peut être restituée de la façon suivante: $\{\alpha(\mathrm{A} / \overline{\mathrm{A}}$, $-1,1), \alpha(\mathrm{A} / \overline{\mathrm{A}},-1,0), \alpha(\mathrm{A} / \overline{\mathrm{A}},-1,-1), \alpha(\mathrm{A} / \overline{\mathrm{A}}, 1,1), \alpha(\mathrm{A} / \overline{\mathrm{A}}, 1,0), \alpha(\mathrm{A} / \overline{\mathrm{A}}, 1,-1)\}$.
} 
entre les pôles canoniques positifs $\left(\mathrm{A}^{+}, \overline{\mathrm{A}}^{+}\right)$, neutres $\left(\mathrm{A}^{0}, \overline{\mathrm{A}}^{0}\right)$ et négatifs $\left(\mathrm{A}^{-}, \overline{\mathrm{A}}^{-}\right)$. Enfin, la classe constituée par les six pôles canoniques peut être dénommée la matrice canonique: $\left\{\mathrm{A}^{+}, \mathrm{A}^{0}, \mathrm{~A}^{-}, \overline{\mathrm{A}}^{+}, \overline{\mathrm{A}}^{0}\right.$, $\left.\overline{\mathrm{A}}^{-}\right\}$.

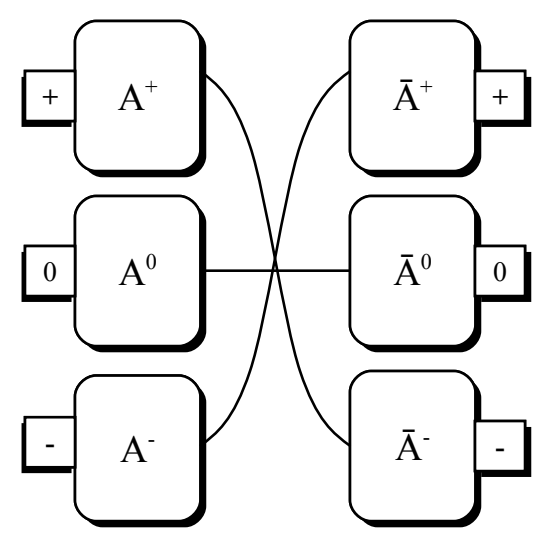

Figure 1

Etudions maintenant la nature des relations existant entre les pôles canoniques d'une matrice donnée. Parmi les combinaisons de relations existant entre les six pôles canoniques $\left(\mathrm{A}^{+}, \mathrm{A}^{0}, \mathrm{~A}^{-}, \overline{\mathrm{A}}^{+}, \overline{\mathrm{A}}^{0}, \overline{\mathrm{A}}^{-}\right)$ d'une même dualité $\mathrm{A} / \overline{\mathrm{A}}$, il convient de mentionner les relations suivantes: dualité, antinomie, complémentarité, corollarité, connexité, anti-connexité. Ainsi, deux pôles canoniques $\alpha_{1}\left(\mathrm{~A} / \overline{\mathrm{A}}, c_{1}, p_{1}\right)$ et $\alpha_{2}\left(\mathrm{~A} / \overline{\mathrm{A}}, c_{2}, p_{2}\right)$ d'une même matrice sont:

(i) duaux si leurs composantes contraires sont opposées et leurs polarités sont neutres

(ii) contraires (ou antinomiques) si leurs composantes contraires sont opposées et leurs polarités sont non neutres et opposées

(iii) complémentaires si leurs composantes contraires sont opposées et leurs polarités sont non neutres et égales

(iv) corollaires si leurs composantes contraires sont égales et leurs polarités sont non neutres et opposées

(v) connexes si leurs composantes contraires sont égales et la valeur absolue de la différence de leurs polarités est égale à 1

(vi) anti-connexes si leurs composantes contraires sont opposées et la valeur absolue de la différence de leurs polarités est égale à 1

Résumons: $\left\{\mathrm{A}^{0}, \overline{\mathrm{A}}^{0}\right\}$ sont duaux, $\left\{\mathrm{A}^{+}, \overline{\mathrm{A}}^{-}\right\}$et $\left\{\mathrm{A}^{-}, \overline{\mathrm{A}}^{+}\right\}$sont contraires, $\left\{\mathrm{A}^{+}, \overline{\mathrm{A}}^{+}\right\}$et $\left\{\mathrm{A}^{-}, \overline{\mathrm{A}}^{-}\right\}$sont complémentaires, $\left\{\mathrm{A}^{+}, \mathrm{A}^{-}\right\}$et $\left\{\overline{\mathrm{A}}^{+}, \overline{\mathrm{A}}^{-}\right\}$sont corollaires, $\left\{\mathrm{A}^{0}, \mathrm{~A}^{+}\right\},\left\{\mathrm{A}^{0}, \mathrm{~A}^{-}\right\},\left\{\overline{\mathrm{A}}^{0}, \overline{\mathrm{A}}^{+}\right\}$et $\left\{\overline{\mathrm{A}}^{0}, \overline{\mathrm{A}}^{-}\right\}$sont connexes, $\left\{\mathrm{A}^{0}, \overline{\mathrm{A}}^{+}\right\},\left\{\mathrm{A}^{0}, \overline{\mathrm{A}}^{-}\right\},\left\{\overline{\mathrm{A}}^{0}, \mathrm{~A}^{+}\right\}$et $\left\{\overline{\mathrm{A}}^{0}, \mathrm{~A}^{-}\right\}$sont anti-connexes.

Il convient maintenant de s'intéresser aux types de relations existant, dans certaines circonstances entre les pôles canoniques de dualités différentes. Définissons préalablement la relation d'englobant. Posons ici qu'un concept $\alpha$ est un englobant pour deux autres concepts $\beta$ et $\chi$ si et seulement si $\alpha=\beta$ $\vee \chi$. Une telle définition capture l'intuition selon laquelle $\alpha$ est le concept minimal dont le contenu sémantique inclut celui de $\beta$ et de $\chi$. Pour prendre un exemple concernant la valeur de vérité, déterminé $e^{2}$ est un englobant pour $\{v r a i, f a u x\}$.

Soient maintenant $\mathrm{A}$ et $\mathrm{E}$ deux matrices dont les pôles canoniques sont respectivement $\left\{\mathrm{A}^{+}, \mathrm{A}^{0}, \mathrm{~A}^{-}\right.$, $\left.\overline{\mathrm{A}}^{+}, \overline{\mathrm{A}}^{0}, \overline{\mathrm{A}}^{-}\right\}$et $\left\{\mathrm{E}^{+}, \mathrm{E}^{0}, \mathrm{E}^{-}, \overline{\mathrm{E}}^{+}, \overline{\mathrm{E}}^{0}, \overline{\mathrm{E}}^{-}\right\}$. Ces matrices sont telles que $\mathrm{E}^{+}, \mathrm{E}^{0}, \mathrm{E}^{-}$sont les englobants respectifs pour $\left\{\mathrm{A}^{+}, \overline{\mathrm{A}}^{+}\right\},\left\{\mathrm{A}^{0}, \overline{\mathrm{A}}^{0}\right\},\left\{\mathrm{A}^{-}, \overline{\mathrm{A}}^{-}\right\}$, c'est-à-dire telles que $\mathrm{E}^{+}=\mathrm{A}^{+} \vee \overline{\mathrm{A}}^{+}, \mathrm{E}^{0}=\mathrm{A}^{0} \vee \overline{\mathrm{A}}^{0}$ et $\mathrm{E}^{-}=$ $\mathrm{A}^{-} \vee \overline{\mathrm{A}}^{-3} .^{3}$

\footnotetext{
${ }^{2}$ La logique trivaluée distingue ainsi trois valeurs de vérité: vrai, faux, indéterminé.

${ }^{3}$ On peut observer que l'une des trois conditions se révèle suffisante. En effet, $E^{+}=A^{+} \vee \bar{A}^{+}$entraine $E^{0}=A^{0} \vee$ $\overline{\mathrm{A}}^{0}$ et $\mathrm{E}^{-}=\mathrm{A}^{-} \vee \overline{\mathrm{A}^{-}} ; \mathrm{E}^{0}=\mathrm{A}^{0} \vee \overline{\mathrm{A}}^{0}$ entraîne $\mathrm{E}^{+}=\mathrm{A}^{+} \vee \overline{\mathrm{A}}^{+}$et $\mathrm{E}^{-}=\mathrm{A}^{-} \vee \overline{\mathrm{A}}^{-} ; \mathrm{E}^{-}=\mathrm{A}^{-} \vee \overline{\mathrm{A}}^{-}$entraîne $\mathrm{E}^{0}=\mathrm{A}^{0} \vee \overline{\mathrm{A}}^{0}$ et $\mathrm{E}^{+}=$ $\mathrm{A}^{+} \vee \overline{\mathrm{A}}^{+}$.
} 


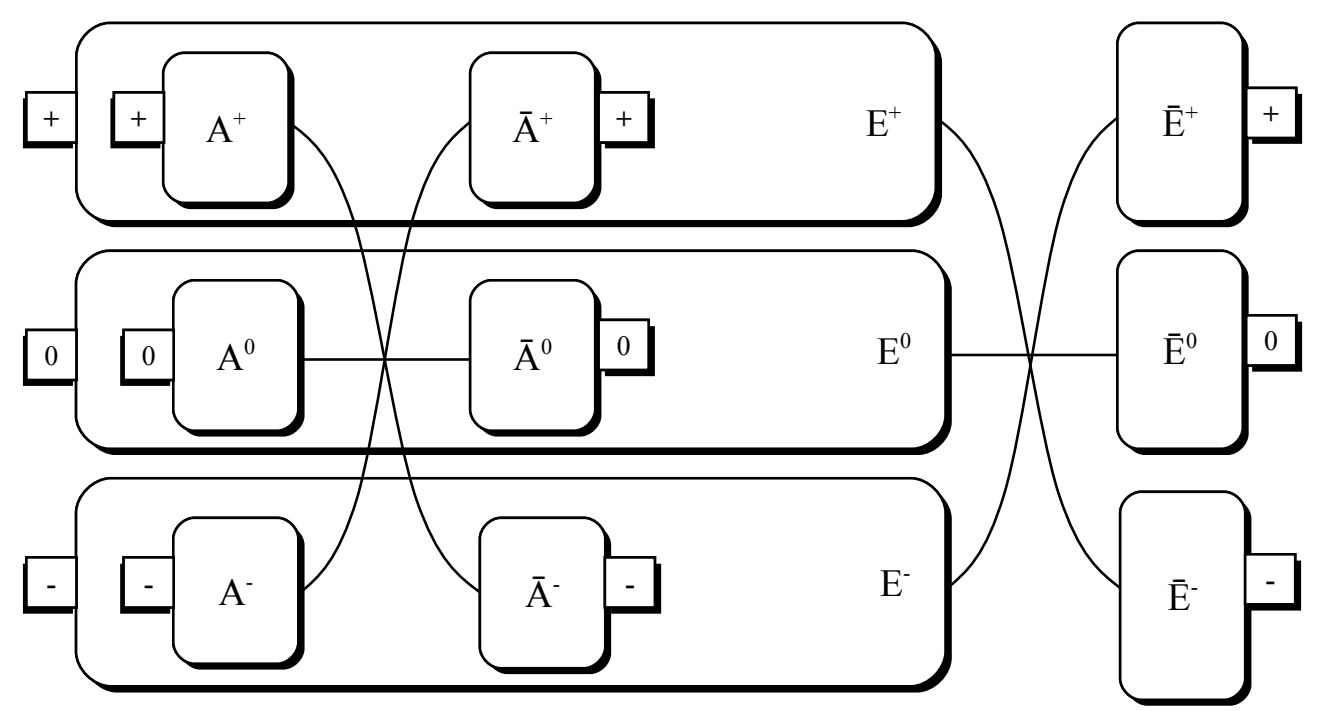

Figure 2

Dénotons cette relation entre les deux matrices par $\mathrm{A}<\mathrm{E}$. On prolonge alors les relations précédemment définies entre les pôles canoniques d'une même matrice, aux relations de même nature existant entre deux matrices présentant les propriétés de $\mathrm{A}$ et de $\mathrm{E}$, c'est-à-dire telles que $\mathrm{A}<\mathrm{E}$. Il en résulte alors de manière directe les relations de 2-dualité, 2-antinomie, 2-complémentarité, 2-anticonnexité. ${ }^{4}$ Ainsi deux pôles canoniques $\alpha_{1}\left(\mathrm{~A} / \overline{\mathrm{A}}, c_{1}, p_{1}\right)$ et $\alpha_{2}\left(\mathrm{E} / \overline{\mathrm{E}}, c_{2}, p_{2}\right)$ de deux matrices différentes sont:

(i') 2-duaux (ou duaux trichotomiques) si leurs polarités sont neutres et si le dual de $\alpha_{2}$ est un englobant pour $\alpha_{1}$

(ii') 2-contraires ${ }^{5}$ (ou contraires trichotomiques) si leurs polarités sont non neutres et opposées et si le contraire de $\alpha_{2}$ est un englobant pour $\alpha_{1}$

(iii') 2-complémentaires (ou complémentaires trichotomiques) si leurs polarités sont non neutres et égales et si le complémentaire de $\alpha_{2}$ est un englobant pour $\alpha_{1}$

(vi') 2-anti-connexes (ou anti-connexes trichotomiques) si la valeur absolue de la différence de leurs polarités est égales à 1 et si l'anti-connexe de $\alpha_{2}$ est un englobant pour $\alpha_{1}$

Résumons: $\left\{\mathrm{A}^{0}, \overline{\mathrm{E}}^{0}\right\}$ et $\left\{\overline{\mathrm{A}}^{0}, \overline{\mathrm{E}}^{0}\right\}$ sont 2-duaux, $\left\{\mathrm{A}^{+}, \overline{\mathrm{E}}^{-}\right\},\left\{\mathrm{A}^{-}, \overline{\mathrm{E}}^{+}\right\},\left\{\overline{\mathrm{A}}^{+}, \overline{\mathrm{E}}^{-}\right\}$et $\left\{\overline{\mathrm{A}}^{-}, \overline{\mathrm{E}}^{+}\right\}$sont 2contraires, $\left\{\mathrm{A}^{+}, \overline{\mathrm{E}}^{+}\right\},\left\{\mathrm{A}^{-}, \overline{\mathrm{E}}^{-}\right\},\left\{\overline{\mathrm{A}}^{+}, \overline{\mathrm{E}}^{+}\right\}$et $\left\{\overline{\mathrm{A}}^{-}, \overline{\mathrm{E}}^{-}\right\}$sont 2-complémentaires, $\left\{\mathrm{A}^{0}, \overline{\mathrm{E}}^{+}\right\},\left\{\mathrm{A}^{0}, \overline{\mathrm{E}}^{-}\right\},\left\{\overline{\mathrm{A}}^{0}\right.$, $\left.\overline{\mathrm{E}}^{+}\right\}$et $\left\{\overline{\mathrm{A}}^{0}, \overline{\mathrm{E}}^{-}\right\}$sont 2 -anti-connexes.

Enfin, il convient de mettre l'accent sur la notion de complément d'un pôle canonique. Soit $\alpha$ un pôle canonique. On dénotera par $\sim \alpha$ son complément, qui correspond sémantiquement à non- $\alpha$. Dans le présent contexte, la notion de complément implique la définition d'un univers de référence. Il convient ainsi de s'intéresser à la notion de complément d'un pôle canonique défini par rapport à la matrice correspondante. Dans ce cas, l'univers de référence est égal à $\left\{\mathrm{A}^{+}, \mathrm{A}^{0}, \mathrm{~A}^{-}, \overline{\mathrm{A}}^{+}, \overline{\mathrm{A}}^{0}, \overline{\mathrm{A}}^{-}\right\}$et $\sim \alpha=\left\{\mathrm{A}^{+}, \mathrm{A}^{0}\right.$, $\left.\mathrm{A}^{-}, \overline{\mathrm{A}}^{+}, \overline{\mathrm{A}}^{0}, \overline{\mathrm{A}}^{-}\right\}-\alpha$. On a alors par exemple $\sim \mathrm{A}^{+}=\left\{\mathrm{A}^{0}, \mathrm{~A}^{-}, \overline{\mathrm{A}}^{+}, \overline{\mathrm{A}}^{0}, \overline{\mathrm{A}}^{-}\right\}$et une définition similaire pour les compléments des autres pôles canoniques de la matrice. Considérons maintenant deux matrices telles que $\mathrm{A}<\mathrm{E}$. Dans ces circonstances, l'univers de référence ${ }^{6}$ est égal à $\left\{\mathrm{A}^{+}, \mathrm{A}^{0}, \mathrm{~A}^{-}, \overline{\mathrm{A}}^{+}, \overline{\mathrm{A}}^{0}, \overline{\mathrm{A}}^{-}, \overline{\mathrm{E}}^{+}\right.$, $\left.\overline{\mathrm{E}}^{0}, \overline{\mathrm{E}}^{-}\right\}$. Appelons-le la 2-matrice de $\alpha$. Il s'ensuit que $\sim \alpha=\left\{\mathrm{A}^{+}, \mathrm{A}^{0}, \mathrm{~A}^{-}, \overline{\mathrm{A}}^{+}, \overline{\mathrm{A}}^{0}, \overline{\mathrm{A}}^{-}, \overline{\mathrm{E}}^{+}, \overline{\mathrm{E}}^{0}, \overline{\mathrm{E}}^{-}\right\}-\alpha$. On a alors la notion de 2-complément d'un pôle canonique $\alpha$, définie par rapport à un univers de référence consistant en la 2-matrice de $\alpha$. Plus généralement, on a la notion de $n$-complément $(n>0)$ d'un pôle canonique par rapport à la $n$-matrice correspondante.

\footnotetext{
${ }^{4}$ On a également la généralisation à $n$ matrices $(n>1)$ de la présente construction avec les relations de $n$-dualité, $n$-antinomie, $n$-complémentarité, $n$-anti-connexité.

${ }^{5} \mathrm{Ou} 2$-antinomiques.

${ }^{6}$ Dans ce contexte, $\mathrm{E}^{+}, \mathrm{E}^{0}$ et $\mathrm{E}^{-}$peuvent être omis sans perte de contenu, étant donné leur nature d'englobants.
} 


\section{Solution}

Avec le dispositif conceptuel approprié désormais en place, nous sommes en mesure de présenter une solution pour le problème AHI. Nous pouvons maintenant analyser le problème à la lumière du cadre conceptuel qui vient d'être décrit. Commençons par analyser les différents concepts de manière détaillée. Le concept d'amour présente une connotation positive. C'est un concept mélioratif qui peut être dénoté par amour ${ }^{+}$. Réciproquement, le concept de haine possède une connotation négative. Il apparaît comme un concept péjoratif qui peut être traduit par haine-. De même, le concept d'indifférence présente également une connotation négative. Il s'agit d'une notion péjorative qui peut être dénotée par indifférence-

A ce stade, une difficulté apparait. En effet, on peut observer que les trois concepts se révèlent mélioratifs ou péjoratifs à un certain degré. Et un tel degré peut varier selon le concept considéré. Par exemple haine- pourrait être péjoratif à un degré 0,95 , alors qu'indifférence- pourrait n'être péjoratif qu'à un degré légèrement inférieur de 0,7 . En outre, un tel degré pourrait également varier d'une culture à une autre culture, ou d'une langue donnée à une autre. Finalement, le degré mélioratif ou péjoratif des trois concepts, pourrait se révéler relatif à une culture donnée.

Cependant, de telles difficultés peuvent être évitées dans le présent contexte, car le raisonnement n'y sera pas basé sur les concepts inhérents à une culture ou à une langue donnée, mais bien sur les concepts canoniques décrits plus haut. Par conséquent, il convient de remplacer nos concepts usuels par les concepts canoniques correspondants. Il peut exister des variations en degrés, ou bien d'une culture à l'autre, au niveau des concepts usuels d'amour, de haine et d'indifférence. Cependant, cela n'affecte pas le présent raisonnement, qui demeure basé sur les concepts canoniques. Le passage des concepts non-canoniques aux concepts canoniques s'effectue comme suit. Soit $d[\alpha]$ le degré péjoratif ou mélioratif d'un concept $\alpha$. Dans ce cas, si $d[\alpha] \in] 0.5$; 1] alors $p[\alpha]=1$; sinon si $d[\alpha] \in[-1$; $-0.5[$ alors $p[\alpha]=-1$. A ce stade, on peut poser légitimement que $p[$ Amour $]=1, p[$ Haine $]=-1$ et $p[$ Indifférence $]=-1 .^{7}$ En conséquence, les trois concepts peuvent désormais être dénotés par Amour ${ }^{+}$, Haine ${ }^{-}$, Indifférence ${ }^{-}$.

Ainsi que cela a été observé plus haut, la relation amour/haine n'est pas problématique en ce sens qu'elle s'identifie avec la relation contraire. Ceci s'applique de manière directe à la relation existant entre les concepts canoniques Amour ${ }^{+} /$Haine $^{-}$. Par conséquent, la matrice correspondante présente la structure suivante: $\left\{\right.$ Amour $^{+}, \mathrm{A}^{0}, \mathrm{~A}^{-}, \overline{\mathrm{A}}^{+}, \overline{\mathrm{A}}^{0}$, Haine $\}$. L'étape suivante est la reconstitution de la matrice complète. Cette dernière tâche peut être accomplie à l'aide de la définition des relations entre les pôles canoniques, à savoir: $\mathrm{A}^{-}$est le corollaire de $\mathrm{Amour}^{+}, \overline{\mathrm{A}}^{+}$est le corollaire de Haine ${ }^{-}, \mathrm{A}^{0}$ est connexe à Amour ${ }^{+}$et anti-connexe à Haine ${ }^{-}, \overline{\mathrm{A}}^{0}$ est connexe à Haine ${ }^{-}$et anti-connexe à Amour ${ }^{+}$. Etant donné ces définitions, on est alors en mesure de reconstituer la matrice canonique correspondante: $\left\{\right.$ Amour $^{+}$, Attraction $^{0}, \mathrm{~A}^{-}$, Défiance $^{+}$, Répulsion $\left.^{0}, \mathrm{Haine}^{-}\right\} .^{8}$

\footnotetext{
${ }^{7}$ Le fait de considérer de manière alternative $p$ [indifférence $]>-0.5$ et donc $p$ [Indifférence] $=0$ conduit également à une solution dans le présent contexte. Dans ce dernier cas, les relations $S$ et $T$ s'assimilent alors toutes deux à l'anti-connexité trichotomique.

${ }^{8}$ Lors du processus de reconstitution de la matrice complète, certains concepts peuvent faire défaut. La raison en est qu'ils ne sont pas lexicalisés dans le langage correspondant. Tel et le cas pour A: Ce dernier concept correspond sémantiquement à attraction inappropriée, excessive.
} 


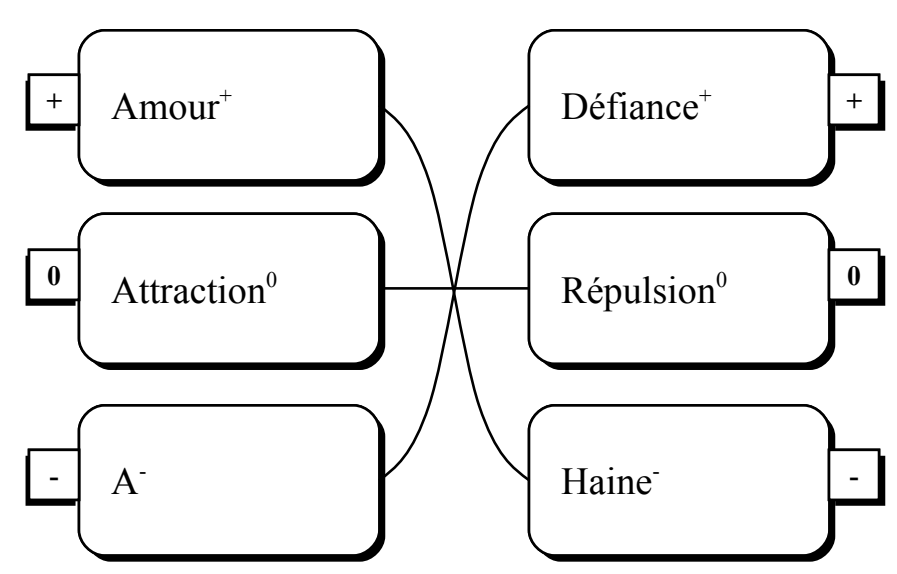

Figure 3

Examinons maintenant le cas du concept d'Indifférence- ${ }^{-}$Un tel concept prend place au sein d'une matrice dont la structure est: $\left\{\mathrm{E}^{+}, \mathrm{E}^{0}, \mathrm{E}^{-}, \overline{\mathrm{E}}^{+}, \overline{\mathrm{E}}^{0}\right.$, Indifférence $\}$. De même que précédemment, il s'avère maintenant nécessaire de reconstituer la matrice complète. Ceci peut être réalisé à l'aide des définitions correspondantes: $\overline{\mathrm{E}}^{+}$est le corollaire d'Indifférence, $\mathrm{E}^{-}$est le complémentaire d'Indifférence', $\mathrm{E}^{+}$est le contraire d'Indifférence', $\overline{\mathrm{E}}^{0}$ est le connexe et le corollaire d'Indifférence-, $\mathrm{E}^{0}$ est l'anti-connexe et le corollaire d'Indifférence- La matrice associée est alors: $\left\{\mathrm{E}^{+}\right.$, Interêt ${ }^{0}, \mathrm{E}^{-}, \mathrm{Flegme}^{+}$, Détachement ${ }^{0}$, Indifférence- $\}$. $^{9}$

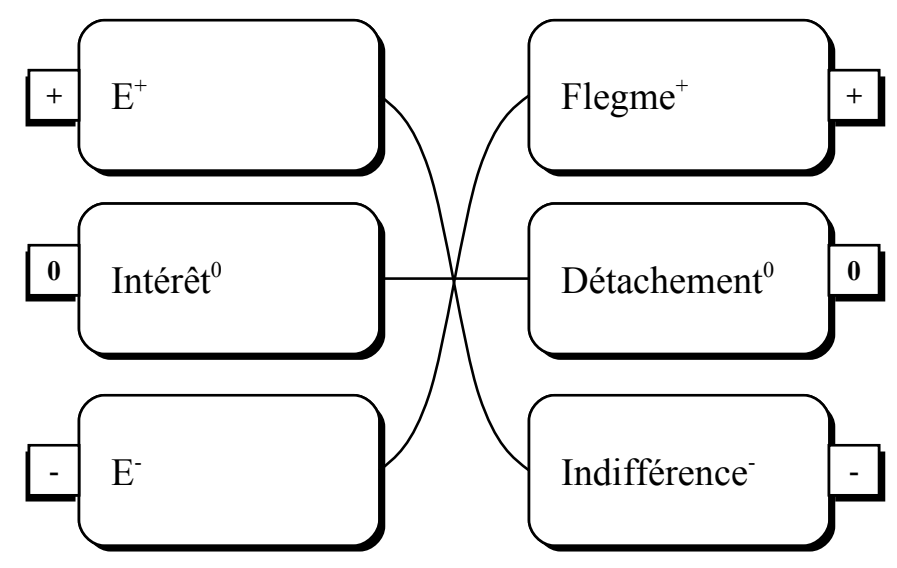

Figure 4

On peut remarquer maintenant que Interêt ${ }^{0}=$ Attraction $^{0} \vee$ Répulsion $^{0}$, c'est-à-dire que Interêt $^{0}$ est un englobant pour Attraction ${ }^{0}$ et Répulsion ${ }^{0}$. Et de même: $\mathrm{E}^{+}=$Amour $^{+} \vee$ Défiance $^{+}$et $\mathrm{E}^{-}=\mathrm{A}^{-} \vee \mathrm{Haine}^{-}$. A ce stade, étant donné que $\left\{\right.$ Amour $^{+}$, Attraction ${ }^{0}, \mathrm{~A}^{-}$, Défiance ${ }^{+}$, Répulsion ${ }^{0}$, Haine $\}<\left\{\mathrm{E}^{+}\right.$, Interêt ${ }^{0}$, $\mathrm{E}^{-}$, Flegme $^{+}$, Détachement ${ }^{0}$, Indifférence $\}$, les relations Amour $^{+} /$Indifférence $^{-}$et Haine ${ }^{-} /$Indifférence $^{-}$en découlent directement. Il résulte en effet des définitions ci-dessus que, d'une part, Amour $^{+}$et Indifférence- sont des contraires trichotomiques et d'autre part, Haine- et Indifférence- sont des complémentaires trichotomiques. A ce stade, on est finalement en mesure de formuler une solution pour le problème AHI:

(i) l'amour est le contraire de la haine

(ii) l'amour est le 2-contraire de l'indifférence

(iii) la haine est le 2-complémentaire de l'indifférence

\footnotetext{
${ }^{9}$ Il semble que les concepts associés à $\mathrm{E}^{+}$et $\mathrm{E}^{-}$ne soient pas lexicalisés. Ils correspondent respectivement à intérêt approprié et à intérêt inapproprié, excessif.
} 
Par conséquent, $R, S, T$ s'identifient respectivement aux relations contraire, contraire trichotomique, complémentaire trichotomique.

\section{Conclusion}

A ce stade, il pourrait être tentant de ne pas considérer l'analyse ci-dessus comme une solution au problème AHI per se. En effet, les concepts d'amour, de haine et d'indifférence semblent constituer des instances d'une classe plus générale de concepts dont les relations sont de même nature. Ceci suggère qu'un même type de solution pourrait être fourni au problème général de la définition des relations entre trois concepts donnés $\alpha, \beta, \chi$ déterminant une trichotomie. Prima facie, certains concepts tels que vrai, faux et indéterminé, tombent également dans le champ de la présente analyse. Toutefois, une telle affirmation doit être envisagée avec prudence. Dans quelle mesure la présente analyse s'applique-t-elle à d'autres trichotomies de concepts? C'est un autre problème qui doit être envisagé, mais dont la résolution dépasse la portée de la présente étude. ${ }^{10}$

\section{Références}

Benzaken, Claude (1991). Systèmes formels. Paris: Masson.

Franceschi, Paul (2002). Une Classe de Concepts. Semiotica, 139, 211-226.

Garlikov, Rick (1998). Understanding, Shallow Thinking, and School.

$<$ http://www.garlikov.com/teaching/Understanding.html> [cité 5 Jan. 2004].

\footnotetext{
${ }^{10}$ Je remercie Rick Garlikov pour des commentaires portant sur une précédente version de cet article.
} 